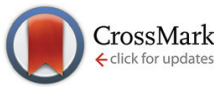

Cite this: Org. Biomol. Chem., 2015, 13,348

Received 2nd October 2014,

Accepted 27th October 2014

DOI: $10.1039 / c 4 o b 02102 a$

www.rsc.org/obc

\section{A peptide topological template for the dispersion of [60]fullerene in water $\dagger$}

\author{
S. Bartocci, D. Mazzier, A. Moretto and M. Mba*
}

\begin{abstract}
Solubilization of [60]fullerene in water is a major challenge for biological and medical applications. To this purpose in this communication we describe for the first time a new dispersing system based on a peptide topological template. The presence of two carbobenzyloxy groups on the peptide side chains allows $\pi-\pi$ interactions with [60]fullerene leading to the formation of stable supramolecular nanocomposites by means of mechanochemical methods. In particular, by high speed vibration milling colloidal dispersions (mean particle diameter $63 \mathrm{~nm}$ ) containing up to $1.3 \mathrm{mg} \mathrm{mL}^{-1}$ of [60]fullerene were obtained. Its presence in water was verified through UV-Vis and MALDI-TOF measurements, while its concentration was determined by thermogravimetric analysis.
\end{abstract}

After the discovery of [60]fullerene, ${ }^{1}$ the third allotropic form of carbon, many scientists were attracted by this fascinating spherical cage and the challenge to study and exploit its properties in different scientific areas. Pristine fullerene is an excellent electron acceptor extensively used in organic solar cells. $^{2,3}$ On the other hand, it also plays an important role in biological and medical applications as an enzyme inhibitor, an antiviral agent or for DNA cleavage and photodynamic therapy. ${ }^{4-8}$ However, the use of the buckyball in these fields has been hampered by its poor solubility in water. On the basis of this, there have been several attempts to overcome the natural hydrophobicity of [60]fullerene. One of the possible strategies is the dispersion of [60]fullerene in the form of aggregates $\left(\mathrm{nC}_{60}\right)$. It has been suggested that colloidal dispersions of $\mathrm{nC}_{60}$ in water may be the main form of fullerene in aquatic systems, ${ }^{9}$ therefore, toxicity and biological studies using these dispersions are of high importance. Stable colloidal dispersions of [60]fullerene can be obtained by the socalled solvent exchange method in which [60]fullerene is trans-

Department of Chemical Sciences, University of Padova, via Marzolo 1, 35135 Padova, Italy.E-mail: miriam.mba@unipd.it

$\dagger$ Electronic supplementary information (ESI) available: Synthesis and full characterization of $\mathbf{1}$ and 2. TGA analysis, MALDI-TOF mass spectra and DLS measurements. See DOI: 10.1039/c4ob02102a ferred from THF, acetone or toluene into water. ${ }^{10-12}$ These methodologies led in general to traces of these highly toxic organic solvents in the aqueous solution; only in one report THF traces could be eliminated by gamma irradiation, ${ }^{13}$ and therefore are not suited for biological or medical applications. Sonication ${ }^{14,15}$ or stirring in water ${ }^{16}$ for long periods of time, weeks or even months, without the addition of any organic solvent has been performed, but as in the solvent-exchange method, the concentration of fullerene achieved in the colloidal solution is very low. Covalent functionalization with suitable hydrophilic substituents leads to water-soluble fullerene derivatives ${ }^{17}$ but disruption of the $\pi$-system may modify its properties and consequently the biological activity. For this reason, non-covalent approaches have been attempted. In particular, complexation of [60]fullerene with host molecules, such as $\gamma$-cyclodextrin, calixarene, cucurbituril and so on, has been proposed for molecular solutions of [60]fullerene. ${ }^{18,19}$ However, these hosts are expensive and difficult to synthesize and, on the other hand, for biological and medical applications the use of biocompatible molecules is highly desirable. Stable dispersions of [60]fullerene have been obtained using biocompatible molecules to stabilize the aggregates, as carbohydrates $^{20}$ and proteins. ${ }^{21}$ In this context, we have recently reported a lysine-based hydrogelator which is able to solubilize [60]fullerene, multi-walled carbon nanotubes (MWCNTs) and graphene in water via a mechanochemical approach. ${ }^{22}$ In this hydrogelator the presence of two 9-fluorenylmethoxycarbonyl (Fmoc) groups ensured $\pi-\pi$ interactions with [60]fullerene and two free amines ensured the solubility in water. Stable colloidal dispersions of [60]fullerene aggregates were obtained at a concentration of $0.8 \mathrm{mg} \mathrm{mL} \mathrm{m}^{-1}$.

In this communication we report the dispersion of [60]fullerene in water through non-covalent functionalization with a cyclic decapeptide 1 (Fig. 1a). Peptide $\mathbf{1}$ has been inspired by the peptide topological templates described by Mutter. ${ }^{23,24}$ These templates present two distinct spatially independent functional domains: four side chains are pointing outwards one face of the cyclic molecule and other two are pointing to 

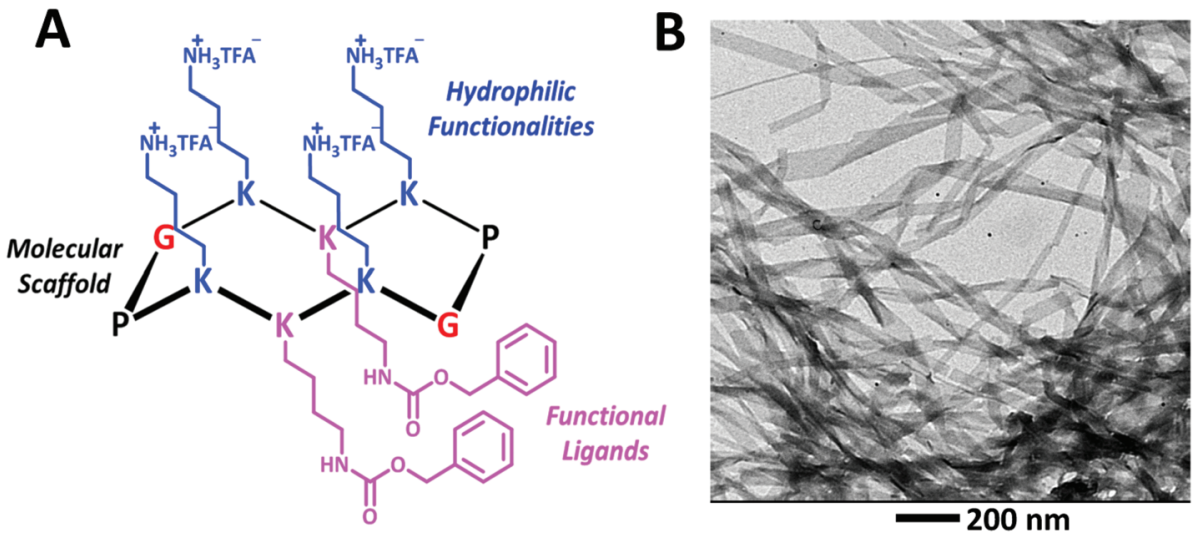

Fig. 1 (a) Structure of 1. (b) TEM images (uranyl stained) of the xerogel obtained from 1.

the opposite face. In our case, one domain will be used to interact with [60]fullerene, and the other domain to control the solubility.

Decapeptide 1 contains six lysines (K) and two Pro-Gly (P-G) $\beta$-turns arranged in a $\beta$ sheet secondary structure (Fig. 1a). The amino group of the side chain is in its free form in the case of the four lysines of one spatial domain, while it is carbobenzyloxy-protected in the case of the two lysines belonging to the spatial domain of the opposite face. The four free amino groups act as hydrophilic functionalities and the two carbobenzyloxy groups ( $\mathrm{Z}$ ) as functional ligands for $\pi-\pi$ interactions with [60]fullerene.

Standard Fmoc mediated solid phase peptide synthesis (SPPS) protocols were used to synthesize the linear decapeptide 2, which was then cyclized using (benzotriazol-1-yloxy) tripyrrolidinophosphonium hexafluorophosphate (pyBOP) as the coupling reagent (for details see ESI $\dagger$ ). Finally, treatment with trifluoroacetic acid (TFA) removed the tert-butyloxycarbonyl (Boc) groups to give $\mathbf{1}$. The structure of 1 was confirmed by NMR, IR and MS (see ESI $\dagger$ ). Peptide 1 proved to be soluble in water at acidic and neutral $\mathrm{pH}$ of up to $20 \mathrm{mg} \mathrm{mL}^{-1}$. At this concentration, when $\mathrm{NaOH}(1 \mathrm{M})$ was added, a transparent stable hydrogel was obtained. Transmission electron microscopy (TEM) images of the xerogel showed the formation of stripes of up to $4 \mu \mathrm{m}$ long (Fig. 1b). We can presume that in these stripes the molecules are arranged in a $\beta$-sheet like hydrogen bond intermolecular network where the hydrophobic part of the molecule is buried inside the supramolecular structures, whereas the hydrophilic part should be in contact with the solvent.

With the aim to compare different methodologies, the $1 / \mathrm{C}_{60}$ nanocomposites were obtained by sonication in water, manual grinding and high speed vibration milling (HSVM). Sonication in water has been traditionally used to obtain inclusion complexes of [60]fullerene with different hosts, ${ }^{25}$ whereas the mechanochemical methodologies are less usual but had demonstrated their utility in covalent and non-covalent functionalization of the highly insoluble carbon nanostructures. ${ }^{26}$ We applied a high-power sonication tip for 1 hour on a suspension of [60]fullerene and $\mathbf{1}$ in $\mathrm{mmQ}$ water $(0.3 \mathrm{~mL}$ $\mathrm{mg}^{-1} \mathrm{C}_{60}$ ). The resulting mixture was then centrifuged first at
$4000 \mathrm{rpm}$ to remove insoluble parts and then at $14000 \mathrm{rpm}$ to eliminate larger aggregates. Finally, it was filtered through a $45 \mu \mathrm{m}$ membrane. In the manual grinding methodology the two solids were grinded in an agate mortar for $20 \mathrm{~min}$, whereas in the case of the HSVM the solids were placed in a stainless-steel milling cup with five stainless-steel mixing balls and milled at a frequency of $30 \mathrm{~Hz}$ for 10 minutes. The resulting solids were suspended in $\mathrm{mmQ}$ water $\left(0.3 \mathrm{~mL} \mathrm{mg}^{-1} \mathrm{C}_{60}\right)$, stirred for $1 \mathrm{~h}$ at room temperature and then treated as described above. In a first set of experiments a $1 / \mathrm{C}_{60}$ ratio $=2: 1$ was used, but most of the [60]fullerene remained insoluble. However, using a $4: 1$ ratio colored solutions were obtained, indicating the presence of fullerene in water. We obtained a reddish-amber solution for the HSVM sample, gold-amber for the manual grinding sample and pale yellow for the sample obtained using sonication (Fig. 2a). The difference in the color intensity indicates qualitatively that the sample obtained from HSVM displays the higher concentration of [60]fullerene.

The presence of fullerene in the solutions was further confirmed by UV-Vis spectroscopy. The absorption spectra of the samples obtained by mechanochemical methods clearly show three new absorption maxima at 262, 341 and $434 \mathrm{~nm}$ plus a weak absorption at $622 \mathrm{~nm}$ (Fig. 2a). This profile is similar to those reported previously for fullerene aggregates $\left(\mathrm{nC}_{60}\right)$ dispersed in water and indicates that the fullerene cage has remained chemically intact during the process. ${ }^{27,28}$ MALDI-TOF experiments showed only the peak of [60]fullerene at $\mathrm{m} / \mathrm{z} 720$ (Fig. 2b and Fig. S20, S21 in the ESI $\dagger$ ), further confirming that no chemical modifications have taken place during the process.

The concentration of [60]fullerene in the solutions was determined by thermogravimetric analysis (TGA) from the relative lyophilized samples. In all cases three main weight losses at 186, 268 and a last one from 545 to $700{ }^{\circ} \mathrm{C}$ (TGA analysis under nitrogen) were observed: the first two losses are attributed to the cyclic decapeptide while the third one includes also a weight loss due to the degradation of fullerene (Fig. 3 and Fig. S18, S19 in the ESI†े). Comparing with the TGA data of 1 alone, the results indicate that we were able to solubilize 1.3, 0.7 and $0.1 \mathrm{mg} \mathrm{mL}^{-1}$ of $\mathrm{C}_{60}$ by HSVM, manual grinding and the sonication procedure, respectively. Therefore HSVM with 

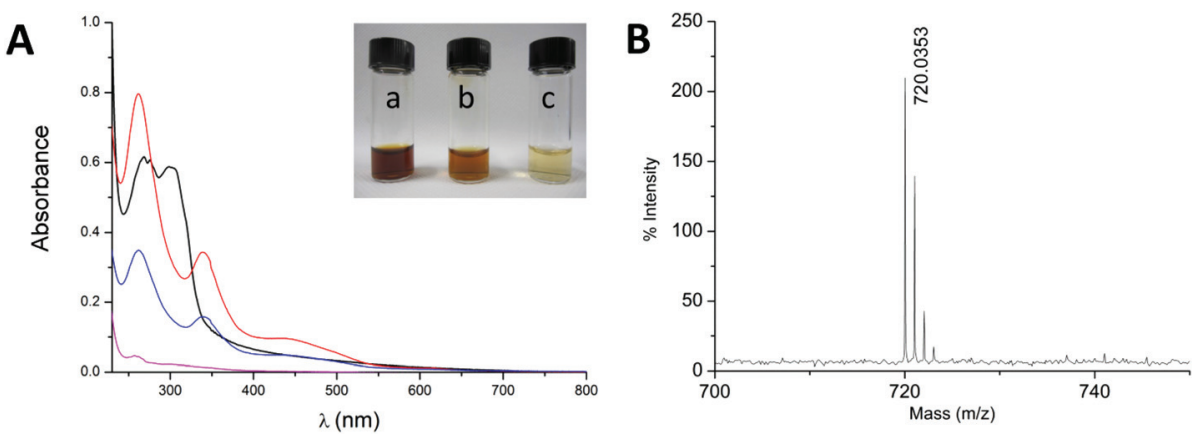

Fig. 2 (a) UV-Vis spectra of aqueous solutions of 1 (black) and $1 / C_{60}$ nanocomposites obtained by HSVM (red), manual grinding (blue) and sonication (pink). Inset: picture of the solutions obtained by HSVM (a), manual grinding (b) and sonication (c). (b) MALDI-TOF mass spectrum of the solution obtained by HSVM.

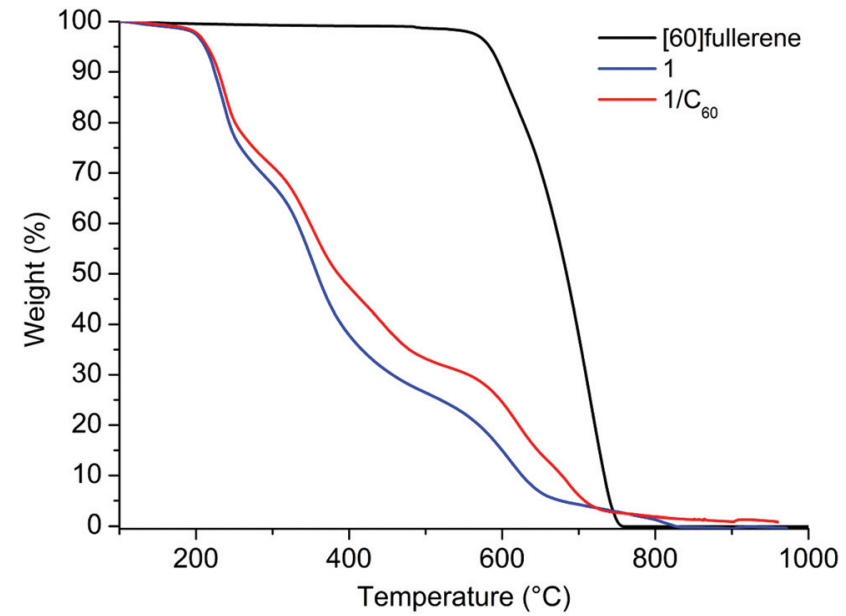

Fig. 3 TGA analysis (under a nitrogen atmosphere) of [60]fullerene, 1 and the $1 / \mathrm{C}_{60}$ solution obtained by HSVM.

peptide 1 allows us to obtain the higher concentration of [60]fullerene and our procedure is far superior to other methods reported to date in the literature for the dispersion of [60]fullerene in the form of aggregates. ${ }^{10-12,20,21}$

The non-covalent interaction between decapeptide 1 and [60]fullerene was confirmed by circular dichroism (CD) spectroscopy. In the $\mathrm{CD}$ spectra weak Cotton effects could be observed in the absorption region of fullerene indicating a transfer of chirality from the chiral decapeptide to the "achiral" fullerene due to a close interaction (Fig. 4). It is most likely that the non-covalent interaction between 1 and [60]fullerene consists mainly of $\pi-\pi$ interactions between the $\pi$-cloud of fullerene and the aromatic $\mathrm{Z}$ group of the peptide.

Particle size of $\mathrm{nC}_{60}$ aggregates has been proved to affect strongly the biological properties of its dispersions. ${ }^{16,29}$ For this reason we investigated the particle size of the dispersions obtained by HSVM by dynamic light scattering (DLS), a wellestablished technique used to determine the size of proteins, micelles and nanoparticles in solution. In Fig. 5 we report our results on the size distribution of the solution obtained by HSVM before and after centrifugation at high speed (14 $000 \mathrm{rpm}$, $5 \mathrm{~min})$. An opaque solution was obtained upon centrifugation

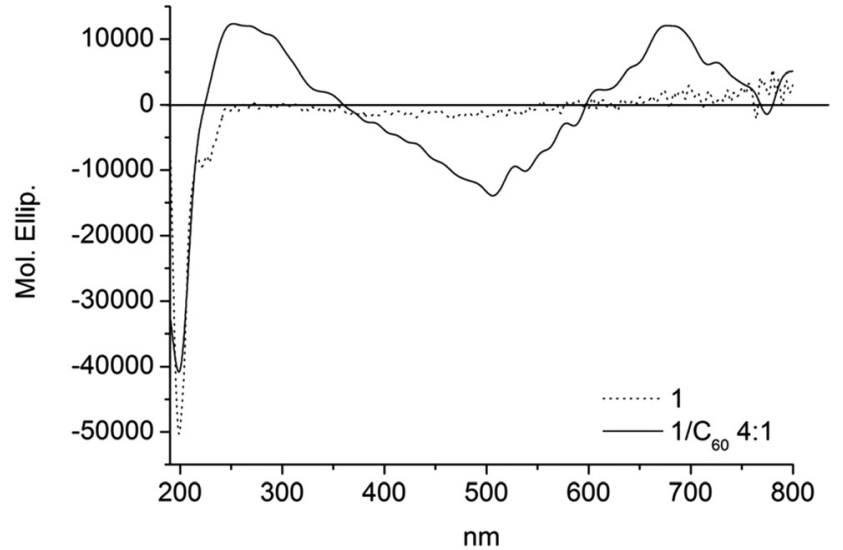

Fig. 4 CD spectra of aqueous solutions of peptide 1 (dotted line) and $1 / \mathrm{C}_{60}$ obtained by HSVM (solid line).

at $4000 \mathrm{rpm}$ that showed two different populations of nanoparticles in the intensity distribution (Fig. 5A). An additional centrifugation at $14000 \mathrm{rpm}$ removed the larger aggregates leading to a transparent solution that showed a single population of aggregates with a mean particle size of $63 \mathrm{~nm}$ that is smaller than those reported in the literature for dispersions of $\mathrm{C}_{60}$ in water obtained by solvent-exchange methods. ${ }^{30}$ Further centrifugation cycles did not cause any change in the DLS measurements. TEM images showed the presence of aggregates of spherical shape with diameters ranging from 20 to $60 \mathrm{~nm}$ (Fig. 6), confirming DLS measurements.

Recently, different authors have shown that in colloidal dispersions of $\mathrm{nC}_{60}$ the spectral properties of the dispersions depend on the particle size of the $\mathrm{nC}_{60}$ aggregates. ${ }^{15,28,31} \mathrm{In}$ particular, the position of the band found at $330-360 \mathrm{~nm}$ is a linear function of the hydrodynamic diameter $\left(Z_{\text {ave }}\right)$. From the different equations reported in the literature, eqn (1) obtained by Deguchi et al. for colloidal dispersions of $\mathrm{nC}_{60}$ with particle size between 30 and $200 \mathrm{~nm}$ stabilized by surfactants ${ }^{15}$ is the most appropriate, since the system used by the authors is the one resembling better the conditions presented in this paper.

$$
\lambda_{\max }(360)=(0.065 \pm 0.009) Z_{\text {ave }}+(337.1 \pm 1.4)
$$




\section{Size distribution by intensity}
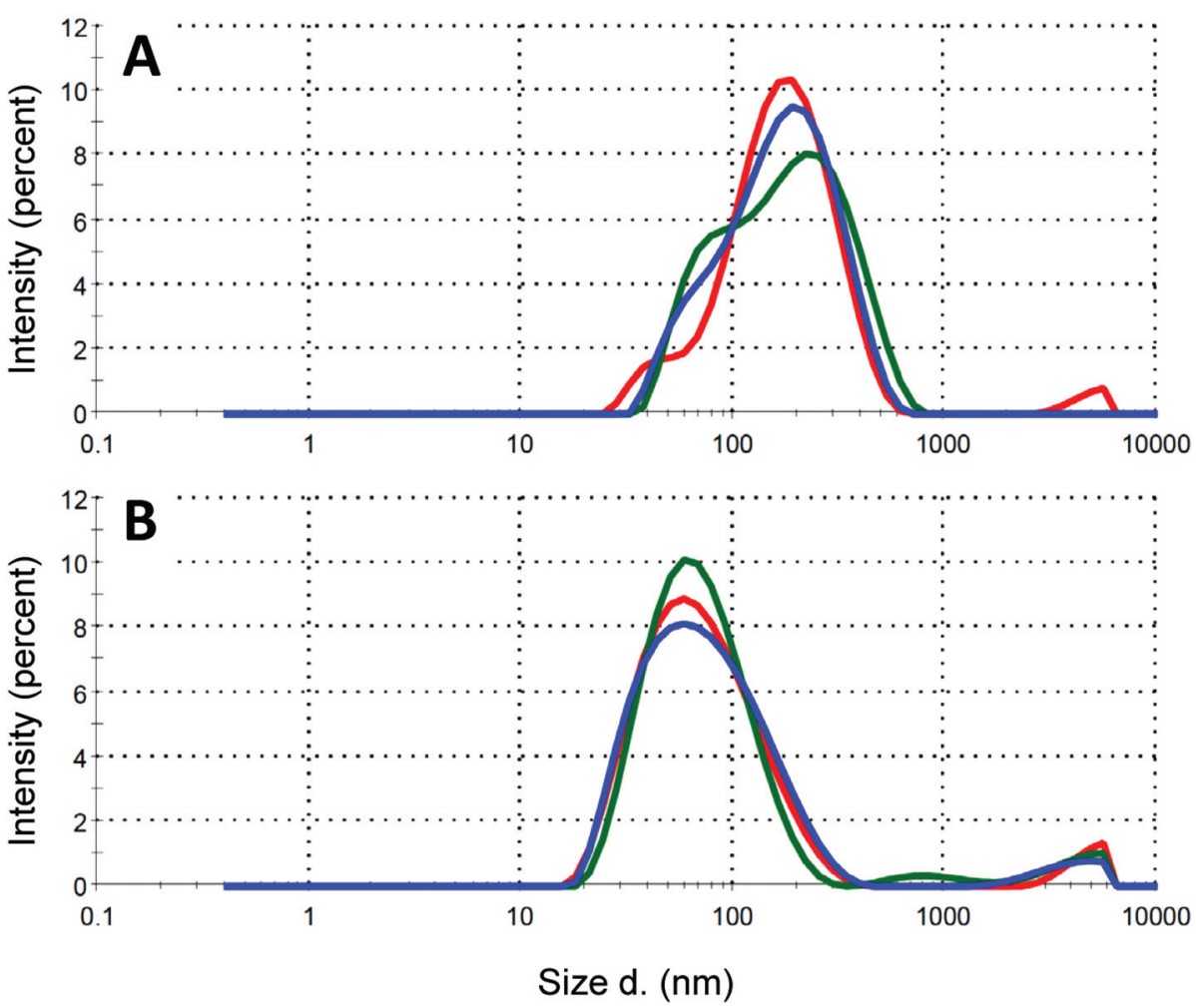

Fig. 5 DLS of the aqueous solutions of $1 / \mathrm{C}_{60}$ nanocomposites obtained by HSVM: (a) upon centrifugation at $4000 \mathrm{rpm}$, (b) upon centrifugation at $14000 \mathrm{rpm}$.

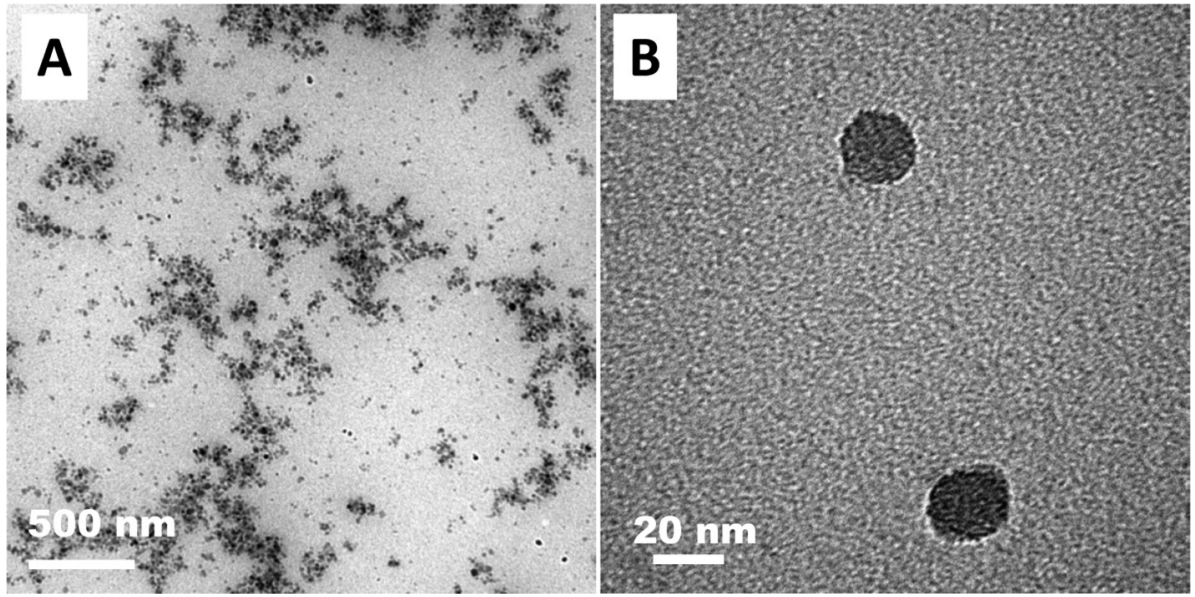

Fig. 6 TEM images of the colloidal dispersion of $1 / C_{60}$ obtained by HSVM.

Introducing in this equation the mean particle size value observed by DLS $(63 \mathrm{~nm})$ a predicted value of $341.1 \mathrm{~nm}$ is obtained for $\lambda_{\max }$ (360), which is in complete agreement with the experimental value found by UV-Vis spectroscopy (341 nm).

The zeta potential measured by DLS for the colloidal dispersion obtained by HSVM had a positive value of $24.7 \mathrm{mV}$, which demonstrates the formation of positively charged surfaces around the $\mathrm{nC}_{60}$ aggregates by peptide 1 .
Another important factor for biological and medical applications is the stability of the dispersion of [60]fullerene aggregates. The high value of zeta potential is the first indication of the high stability of the dispersion, as at large zeta potential values the electrostatic repulsions should prevent further aggregation of the $\mathrm{nC}_{60}$ nanoparticles. We were delighted to find that solutions were stable for months (at least two months) on storage at room temperature and no precipitation 
of any solids was observed. The mean particle size of the aged solutions found by DLS measurements was similar to the one encountered for the freshly prepared samples, hence no aggregation of the nanoparticles occurred on time. Moreover, the solutions could be lyophilized and the resulting powders could be stored at room temperature for weeks and then re-dissolved in water without aggregation or precipitation and no significant changes were observed in DLS measurements (see Fig. S22 in the ESI $\dagger$ ). Dispersions were stable in physiological solution, and no precipitation was observed in the presence of $1 \% \mathrm{NaCl}$ even after $24 \mathrm{~h}$ of the addition of salt. Due to the presence of four charged ammonium groups we expected the solutions to be sensitive to $\mathrm{pH}$ changes. The dispersions were stable at neutral and acidic $\mathrm{pH}$. In contrast, addition of the base leads to the precipitation and not to the hydrogel formation as observed for peptide 1 alone. This is probably due to a concentration lower than $20 \mathrm{mg} \mathrm{mL}^{-1}$ of peptide in the $1 / \mathrm{C}_{60}$ solutions.

In conclusion, we have synthesized a cyclic decapeptide $\mathbf{1}$ and, using high speed vibration milling, we were able to obtain efficiently water soluble $\mathbf{1} / \mathrm{C}_{60}$ nanocomposites without the aid of organic solvents. Highly homogeneous dispersions of [60]fullerene aggregates in water with concentrations of up to $1.3 \mathrm{mg} \mathrm{mL} \mathrm{m}^{-1}$ were achieved when a $1 / \mathrm{C}_{60}$ ratio $4: 1$ was used. We are currently working on the use of the colloidal dispersions of [60]fullerene obtained in this work in biological and medical applications, as appropriate functionalization of the topological template could allow the introduction of recognition sites or any other functionality of interest.

\section{Acknowledgements}

The authors are grateful to Dr F. Caicci (U. Padova) for TEM data recording. The University of Padova (PRAT CPDA114475, PRAT CPDA119117), and MIUR (FIRB project NANOSOLAR RBAP11C58Y) are acknowledged for financial support. S. B. acknowledges the University of Padova for a postdoctoral grant (assegno di ricerca junior CPDR131937).

\section{References}

1 H. W. Kroto, J. R. Heath, S. C. O'Brien, R. F. Curl and R. E. Smalley, Nature, 1985, 318, 162-163.

2 B. C. Thompson and J. M. J. Fréchet, Angew. Chem., Int. Ed., 2008, 47, 58-77.

3 P. Hudhomme and J. Cousseau, in Fullerenes: Principles and Applications (2), The Royal Society of Chemistry, 2012, pp. 416-461.

4 R. Bakry, R. M. Vallant, M. Najam-Ul-Haq, M. Rainer, Z. Szabo, C. W. Huck and G. K. Bonn, Int. J. Nanomed., 2007, 2, 639-649.

5 A. Bianco and T. Da Ros, in Fullerenes: Principles and Applications (2), The Royal Society of Chemistry, 2012, pp. 507-545.

6 R. Partha and J. L. Conyers, Int. J. Nanomed., 2009, 4, 261-275.

7 A. W. Jensen, S. R. Wilson and D. I. Schuster, Bioorg. Med. Chem., 1996, 4, 767-779.
8 S. Bosi, T. Da Ros, G. Spalluto and M. Prato, Eur. J. Med. Chem., 2003, 38, 913-923.

9 L. K. Duncan, J. R. Jinschek and P. J. Vikesland, Environ. Sci. Technol., 2007, 41, 173-178.

10 W. A. Scrivens, J. M. Tour, K. E. Creek and L. Pirisi, J. Am. Chem. Soc., 1994, 116, 4517-4518.

11 G. V. Andrievsky, M. V. Kosevich, O. M. Vovk, V. S. Shelkovsky and L. A. Vashchenko, J. Chem. Soc., Chem. Commun., 1995, 1281-1282.

12 S. Deguchi, R. G. Alargova and K. Tsujii, Langmuir, 2001, 17, 6013-6017.

13 A. Isakovic, Z. Markovic, N. Nikolic, B. Todorovic-Markovic, S. Vranjes-Djuric, L. Harhaji, N. Raicevic, N. Romcevic, D. Vasiljevic-Radovic, M. Dramicanin and V. Trajkpvic, Biomaterials, 2006, 27, 5049-5058.

14 T. Benn, B. G. Pycke, P. Herckes, P. Westerhoff and R. Halden, Anal. Bioanal. Chem., 2011, 399, 1631-1639.

15 S. Deguchi, S.-a. Mukai, T. Yamazaki, M. Tsudome and K. Horikoshi, J. Phys. Chem. C, 2009, 114, 849-856.

16 D. Y. Lyon, L. K. Adams, J. C. Falkner and P. J. J. Alvarez, Environ. Sci. Technol., 2006, 40, 4360-4366.

17 T. Da Ros, M. Prato, F. Novello, M. Maggini and E. Banfi, J. Org. Chem., 1996, 61, 9070-9072.

18 A. Ikeda, A. Hirata, M. Ishikawa, J.-i. Kikuchi, S. Mieda and W. Shinoda, Org. Biomol. Chem., 2013, 11, 7843-7851.

19 K. Nobusawa, D. Payra and M. Naito, Chem. Commun., 2014, 50, 8339-8342.

20 V. I. Bhoi, S. Kumar and C. N. Murthy, Carbohydr. Res., 2012, 359, 120-127.

21 H. Wu, L. Lin, P. Wang, S. Jiang, Z. Dai and X. Zou, Chem. Commun., 2011, 47, 10659-10661.

22 M. Mba, A. I. Jiménez and A. Moretto, Chem. - Eur. J., 2014, 20, 3888-3893.

23 S. Peluso, T. Ruckle, C. Lehmann, M. Mutter, C. Peggion and M. Crisma, ChemBioChem, 2001, 2, 432-437.

24 G. V. Nikiforovich, M. Mutter and C. Lehmann, Biopolymers, 1999, 50, 361-372.

25 P. Hammershøj, P. H. H. Bomans, R. Lakshminarayanan, J. Fock, S. H. Jensen, T. S. Jespersen, T. Brock-Nannestad, T. Hassenkam, J. Nygård, N. A. J. M. Sommerdijk, K. Kilså, T. Bjørnholm and J. B. Christensen, Chem. - Eur. J., 2012, 18, 8716-8723.

26 S.-E. Zhu, F. Li and G.-W. Wang, Chem. Soc. Rev., 2013, 42, $7535-7570$.

27 P. Scharff, K. Risch, L. Carta-Abelmann, I. M. Dmytruk, M. M. Bilyi, O. A. Golub, A. V. Khavryuchenko, E. V. Buzaneva, V. L. Aksenov, M. V. Avdeev, Y. I. Prylutskyy and S. S. Durov, Carbon, 2004, 42, 1203-1206.

28 X. J. Chang and P. J. Vikesland, Environ. Sci. Technol., 2011, 45, 9967-9974.

29 A. Dhawan, J. S. Taurozzi, A. K. Pandey, W. Shan, S. M. Miller, S. A. Hashsham and V. V. Tarabara, Environ. Sci. Technol., 2006, 40, 7394-7401.

30 J. A. Brant, J. Labille, J.-Y. Bottero and M. R. Wiesner, Langmuir, 2006, 22, 3878-3885.

31 H. Kato, A. Nakamura, K. Takahashi and S. Kinugasa, Phys. Chem. Chem. Phys., 2009, 11, 4946-4948. 